\title{
COMMUTATIVE SEMIGROUP AMALGAMS
}

\author{
J. M. HOWIE
}

(Received 8 May 1967)

In the terminology of J. R. Isbell [5], an element $d$ of a semigroup $S$ is dominated by a subsemigroup $U$ of $S$ if, for an arbitrary semigroup $X$ and arbitrary homomorphisms $\alpha, \beta$ from $S$ into $X, \alpha(u)=\beta(u)$ for every $u$ in $U$ implies $\alpha(d)=\beta(d)$. The set of elements of $S$ dominated by $U$ is a subsemigroup of $S$ containing $U$ and is called the dominion of $U$. It was shown by Isbell that if one takes two disjoint isomorphic copies $S^{+}, S^{-}$of $S$ and forms their amalgamated free product $S^{+} *{ }_{U} S^{-}$, that is to say, the quotient of the free product $S^{+} * S^{-}$by the congruence $\rho$ generated by

$$
\Re=\left\{\left(u^{+}, u^{-}\right): u \in U\right\},
$$

$\left(u^{+}\right.$and $u^{-}$being the images of $u$ in $S^{+}, S^{-}$respectively) then the homomorphisms $\mu^{+}: S \rightarrow S^{+} *{ }_{U} S^{-}, \mu^{-}: S \rightarrow S^{+}{ }^{*} S^{-}$defined by

$$
\mu^{+}(s)=s^{+} \rho, \mu^{-}(s)=s^{-} \rho
$$

are one-one. Moreover, $\mu^{+}(s)=\mu^{-}(t)$ only if $s=t$, and $\mu^{+}(s)=\mu^{-}(s)$ if and only if $s$ is in the dominion of $U$. In other words, the two natural copies of $S$ in $S^{+} * S_{U} S^{-}$intersect precisely in the dominion of $U$. Thus, in particular, and in the terminology of $[3]$, the amalgam $\left[S^{+}, S^{-} ; U\right]$ is embeddable if and only if $U$ is self-dominating (that is to say, its own dominion) in $S$.

One obvious question left unanswered in [5] and in the sequel [4] is whether an amalgam $[S, T ; U]$ is necessarily embeddable if $U$ is selfdominating both in $S$ and in $T$. A (commutative) example is given in $\S 2$ to show that this need not be so. Another example shows that an amalgam $[S, T ; U]$ may be embeddable even if $U$ is not self-dominating either in $S$ or in $T$.

In the preliminary section it is shown (Theorem 1.3) that amalgamated free products are associative, and this is then used to show that if $U$ is a semigroup such that every amalgam $[S, T ; U]$ of two semigroups with $U$ as "core" is embeddable, then every amalgam $\left[S_{i} ; U\right]$ of arbitrarily many semigroups with $U$ as core is embeddable. This result leads to considerable simplification in the proofs in $\S 3$.

Isbell [5] calls a semigroup absolutely closed if it is self-dominating wherever embedded. For the purposes of this paper, concerned principally 
as it is with commutative semigroups, the term "absolutely" may be misleading, since it does not seem to be obvious that a commutative semigroup which is always self-dominating when embedded in a commutative semigroup is necessarily self-dominating when embedded in a non-commutative semigroup. It would perhaps be preferable to refer to a semigroup belonging to a subcategory $\boldsymbol{K}$ of the category $\boldsymbol{S g}$ of semigroups as being $\boldsymbol{K}$-closed if it is its own dominion whenever it is embedded in a semigroup in $K$. It may, therefore, be possible for a semigroup in the category $\mathbf{C S g}$ of commutative semigroups to be $\boldsymbol{C S} \boldsymbol{g}$-closed but not $\boldsymbol{S} \boldsymbol{g}$-closed. No example, however, is known to me. Examples are not hard to find if $\boldsymbol{C S g}$ is replaced by a more trivial subcategory of $\boldsymbol{S} \boldsymbol{g}$. For example, if we consider the subcategory $\boldsymbol{R} \boldsymbol{B}$ of rectangular bands $([1], \S 1.8)$, it is fairly easy to show that any rectangular band is $\boldsymbol{R B}$-closed; however, it need not be $\boldsymbol{S} \boldsymbol{g}$-closed, as is shown by $([4]$, Theorem 2.9$)$.

A commutative semigroup $U$ will be called $\boldsymbol{C S g}$-amalgamable if the amalgam $\left[S_{i} ; U\right]$ is embeddable (in a commutative semigroup) for any family $\left\{S_{i}: i \in I\right\}$ of commutative semigroups containing $U$. It is known ([4], Theorems 2.3 and 2.6) that commutative regular semigroups and commutative totally division-ordered semigroups are $\mathbf{C S g}$-closed. In $\S \mathbf{3}$ it is shown that they are in fact $\boldsymbol{C S g}$-amalgamable. It remains an open question whether a commutative semigroup can be $\boldsymbol{C S g}$-closed but not $\boldsymbol{C S g}$-amalgamable.

\section{Preliminaries}

Various basic definitions and results of semigroup theory, all to be found in Clifford and Preston [1], will be used without comment. Section 1.5, on congruences, factor groupoids and homomorphisms, will be particularly relevant. However, unlike Clifford and Preston, I shall write mappings on the left, and also consider relations on a set $S$ as subsets of the Cartesian square $S \times S$.

The language and the methods used in this section are to a large extent those of category theory and the results are in particular valid in both $\boldsymbol{S g}$ (semigroups and homomorphisms) and $\boldsymbol{C S g}$ (commutative semigroups and homomorphisms), the two categories that principally concern us here. Following Mitchell ([6], § 1.7), we call a commutative diagram

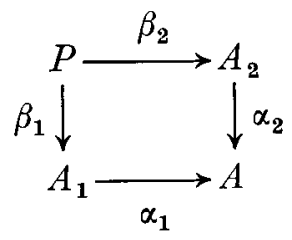


a pullback for $\alpha_{1}$ and $\alpha_{2}$ if for every pair of homomorphisms $\beta_{1}^{\prime}: P^{\prime} \rightarrow A_{1}$ and $\beta_{2}^{\prime}: P^{\prime} \rightarrow A_{2}$ such that $\alpha_{1} \beta_{1}^{\prime}=\alpha_{2} \beta_{2}^{\prime}$ there exists a unique homomorphism $\gamma: P^{\prime} \rightarrow P$ such that $\beta_{1}^{\prime}=\beta_{1} \gamma$ and $\beta_{2}^{\prime}=\beta_{2} \gamma$. Dually, a commutative diagram

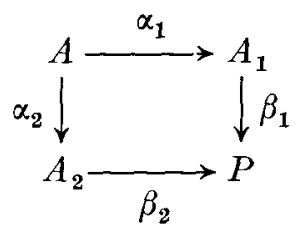

is called a pushout for $\alpha_{1}$ and $\alpha_{2}$ if for every pair of homomorphisms $\beta_{1}^{\prime}: A_{1} \rightarrow P^{\prime}$ and $\beta_{2}^{\prime}: A_{2} \rightarrow P^{\prime}$ such that $\beta_{1}^{\prime} \alpha_{1}=\beta_{2}^{\prime} \alpha_{2}$ there exists a unique homomorphism $\gamma: P \rightarrow P^{\prime}$ such that $\beta_{1}^{\prime}=\gamma \beta_{1}$ and $\beta_{2}^{\prime}=\gamma \beta_{2}$. We shall actually require a (possibly) infinite version of this latter definition: the commutative diagram

$$
\left\{A \stackrel{\alpha_{i}}{\rightarrow} A_{i} \stackrel{\beta_{i}}{\rightarrow} P\right\}_{i \in I}
$$

is a pushout for $\left\{\alpha_{i}\right\}_{i \in I}$ if, for every family $\left\{\beta_{i}^{\prime}: A_{i} \rightarrow P^{\prime}\right\}_{i \in I}$ of homomorphisms such that the diagram

$$
\left\{A \stackrel{\alpha_{i}}{\rightarrow} A_{i} \stackrel{\beta^{\prime} i}{\rightarrow} P^{\prime}\right\}_{i \in I}
$$

commutes, there exists a unique homomorphism $\gamma: P \rightarrow P^{\prime}$ such that $\beta_{i}^{\prime}=\gamma \beta_{i}$ for every $i$ in $I$.

As in $[3]$, a (semigroup) amalgam consists of a semigroup $U$ together with a family $\left\{S_{i}: i \in I\right\}$ of semigroups and a family $\left\{\phi_{i}: i \in I\right\}$ of monomorphisms $\varphi_{i}: U \rightarrow S_{i}$. We denote the amalgam by

$$
\mathfrak{A}=\left[\left\{S_{i}: i \in I\right\} ; U ;\left\{\varphi_{i}: i \in I\right\}\right],
$$

or by $\left[S_{i} ; U ; \varphi_{i}\right]$ or just $\left[S_{i} ; U\right]$ if the context allows. The semigroup $U$ will be called the core of the amalgam $\mathfrak{A}$.

The free product $P=\Pi_{U}^{*}\left\{S_{i}: i \in I\right\}$ of the amalgam $\mathfrak{A}$ is defined as $F / \rho$, where $F$ is the coproduct (in the terminology of Mitchell [6]) of the objects $S_{i}$ (that is to say, their free product in $\boldsymbol{S g}$ and their "rirect product" in the sense of [3] in $\boldsymbol{C S g}$ ) and $\rho$ is the congruence on $F$ generated by

$$
\Re=\left\{\left(\varphi_{i}(u), \varphi_{j}(u)\right): u \in U, i, j \in I\right\} .
$$

For each $i$ in $I$ there is a homomorphism $\mu_{i}: S_{i} \rightarrow P$ defined by

and the diagram

$$
\mu_{i}(x)=x \rho \quad\left(x \in S_{i}\right)
$$

$$
\left\{U \stackrel{\varphi_{i}}{\rightarrow} S_{i} \stackrel{\mu_{i}}{\rightarrow} P\right\}_{i \in I}
$$

is commutative. In fact, the diagram (1) is a pushout for $\left\{\varphi_{i}\right\}_{i \in I}$, and $P$ is 
determined to within isomorphism by this property. This has been shown (in different language) by B. H. Neumann ([7], p. 505) for the category of groups; it is not hard to modify his proof to deal with the categories $\mathrm{Sg}$ and $\mathrm{CSg}$.

The amalgam $\mathfrak{A}$ is said to be embeddable if for some semigroup $T$ there exists for each $i$ in $I$ a monomorphism $\lambda_{i}: S_{i} \rightarrow T$ such that the diagram

$$
\left\{U \stackrel{\varphi_{i}}{\rightarrow} S_{i} \stackrel{\lambda_{i}}{\rightarrow} T\right\}_{i \in I}
$$

is commutative and such that

$$
\lambda_{i}\left(S_{i}\right) \cap \lambda_{j}\left(S_{j}\right)=\lambda(U)
$$

if $i, j$ are distinct elements of $I$, where $\lambda: U \rightarrow T$ is the common value of $\lambda_{i} \varphi_{i}(i \in I)$. We say that the amalgam $\mathfrak{A}$ is embedded in $T$.

From the pushout property of the diagram (1) we can deduce

Theorem 1.1. An amalgam

$$
\mathfrak{A}=\left[\left\{S_{i}: i \in I\right\} ; U ;\left\{p_{i}: i \in I\right\}\right]
$$

is embeddable if and only if

(i) $\mu_{i}$ is a monomorphism for each $i$;

(ii) $\mu_{i}\left(S_{i}\right) \cap \mu_{j}\left(S_{j}\right)=\mu(U)$ if $i \neq j$, where $\mu: U \rightarrow P$ is equal to $\varphi_{i} \mu_{i}$ for some (and therefore every) $i$ in $I$.

Thus, informally, the amalgam is embeddable if and only if it is embeddable in $P$.

Using ([6], Proposition 8.1) we obtain

THEOREM 1.2. The amalgam $\mathfrak{A}$ is embedded in $T$ if and only if there exists a monomorphism $\lambda_{i}: S_{i} \rightarrow T$ for each $i$ in $I$ such that the diagram

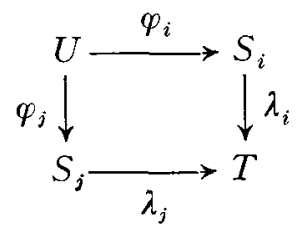

is commutative and is a pullback for $\lambda_{i}$ and $\lambda_{j}$ for every pair $i$, $j$ of distinct elements of $I$.

We now derive a general associativity property for amalgamated free products.

Theorem 1.3. Let $\left[\left\{S_{i}: i \in I\right\} ; U ;\left\{\varphi_{i}: i \in I\right\}\right]$ be an amalgam. Suppose that the index set $I$ is partitioned into disjoint subsets $J_{k}(k \in K)$ and that the amalgam $\left[\left\{S_{j}: j \in J_{k}\right\} ; U ;\left\{\varphi_{j}: j \in J_{k}\right\}\right]$ is embeddable for each $k$. Let 
$P_{k}=\Pi_{U}^{*}\left\{S_{j}: j \in J_{k}\right\}$. Let $\mu_{k}\left(=\mu_{j} \varphi_{j}\right.$ for every $j$ in $\left.J_{k}\right)$ be the natural monomorphism from $U$ into $P_{k}$ and suppose that the amalgam

$$
\left[\left\{P_{k}: k \in K\right\} ; U ;\left\{\mu_{k}: k \in K\right\}\right]
$$

is embeddable. Then the amalgam

is embeddable, and

$$
\left[\left\{S_{i}: i \in I\right\} ; U ;\left\{\varphi_{i}: i \in I\right\}\right]
$$

$$
\Pi_{U}^{*}\left\{S_{i}: i \in I\right\} \cong \Pi_{U}^{*}\left\{\Pi_{U}^{*}\left\{S_{j}: j \in J_{k}\right\}: k \in K\right\} .
$$

Proof. By virtue of Theorems 1.1 and 1.2 we can assume that for every $k$ in $K$ and every $j, j^{\prime} \in J_{k}$, the diagram

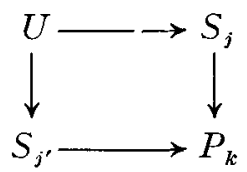

is a pullback for the natural monomorphisms $S_{j} \rightarrow P_{k}, S_{j^{\prime}} \rightarrow P_{k}$. Also, if $P=\Pi_{U}^{*}\left\{P_{k}: k \in K\right\}$, then for every $k, l$ in $K$,

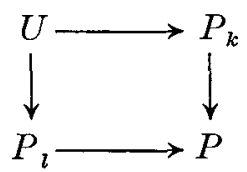

is a pullback for the natural monomorphisms $P_{k} \rightarrow P, P_{l} \rightarrow P$. We certainly have a (perhaps infinite) commutative diagram

$$
\left\{U \rightarrow S_{i} \rightarrow P\right\}_{i \in I},
$$

where, for each $i$, the monomorphism $S_{i} \rightarrow P$ is equal to $S_{i} \rightarrow P_{k} \rightarrow P$ for the appropriate $k$. To prove the theorem we must show that (1) is a pushout for $\left\{U \rightarrow S_{i}\right\}_{i \in I}$ and that for every $i, j$ in $I$ such that $i \neq j$,

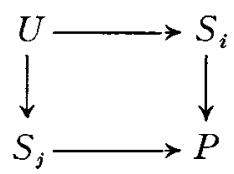

is a pullback for $S_{i} \rightarrow P$ and $S_{j} \rightarrow P$.

To establish the first of these properties, suppose that for some $P^{\prime}$ there exist homomorphisms $S_{i} \rightarrow P^{\prime}$ such that

$$
\left\{U \rightarrow S_{i} \rightarrow P^{\prime}\right\}_{i \in I}
$$

is a commutative diagram. Then by the pushout property of the diagram 


$$
\left\{U \rightarrow S_{j} \rightarrow P_{k}\right\}_{j \in J_{k}}
$$

there exists for each $k$ in $K$ a unique $P_{k} \rightarrow P^{\prime}$ such that

$$
U \rightarrow S_{j} \rightarrow P^{\prime}=U \rightarrow S_{j} \rightarrow P_{k} \rightarrow P^{\prime}
$$

for every $j$ in $J_{k}$. By the commutativity of the diagram (6), it follows that

$$
\left\{U \rightarrow P_{k} \rightarrow P^{\prime}\right\}_{k \in K}
$$

is a commutative diagram, where $U \rightarrow P_{k}$ is taken as $U \rightarrow S_{j} \rightarrow P_{k}$ for some (and therefore every) $j$ in $J_{k}$.

By the pushout property of the diagram

$$
\left\{U \rightarrow P_{k} \rightarrow P\right\}_{k \in K},
$$

there exists a unique $P \rightarrow P^{\prime}$ such that

$$
U \rightarrow P_{k} \rightarrow P^{\prime}=U \rightarrow P_{k} \rightarrow P \rightarrow P^{\prime}
$$

for every $k$ in $K$. Thus

$$
U \rightarrow S_{j} \rightarrow P_{k} \rightarrow P^{\prime}=U \rightarrow S_{j} \rightarrow P_{k} \rightarrow P \rightarrow P^{\prime}
$$

for every $k$ in $K$ and every $j$ in $J_{k}$ and so, using (7) and the commutativity of the diagram (8), we deduce that

$$
U \rightarrow S_{i} \rightarrow P^{\prime}=U \rightarrow S_{i} \rightarrow P \rightarrow P^{\prime}
$$

for every $i$ in $I$. Moreover, the uniqueness of $P \rightarrow P^{\prime}$ in (9) guarantees its uniqueness in $(10)$; thus $\left\{U \rightarrow S_{i} \rightarrow P\right\}_{i \in I}$ is a pushout for $\left\{U \rightarrow S_{i}\right\}_{i \in I}$ as asserted.

For the other result we must consider two cases separately: (i) $i, j \in J_{k}$; (ii) $i \in J_{k}, j \in J_{l}, k \neq l$.

In case (i) we have that both $S_{i} \rightarrow P$ and $S_{j} \rightarrow P$ factor through $P_{k}$. If for some $U^{\prime}$ there exist $U^{\prime} \rightarrow S_{i}, U^{\prime} \rightarrow S_{j}$ such that

it follows that

$$
U^{\prime} \rightarrow S_{i} \rightarrow P=U^{\prime} \rightarrow S_{j} \rightarrow P,
$$

$$
U^{\prime} \rightarrow S_{i} \rightarrow P_{k} \rightarrow P=U^{\prime} \rightarrow S_{j} \rightarrow P_{k} \rightarrow P .
$$

Since $P_{k} \rightarrow P$ is a monomorphism, it follows that

$$
U^{\prime} \rightarrow S_{i} \rightarrow P_{k}=U^{\prime} \rightarrow S_{j} \rightarrow P_{k} ;
$$

hence, by the pullback property of the diagram (3), there exists a unique $U^{\prime} \rightarrow U$ such that the diagram 


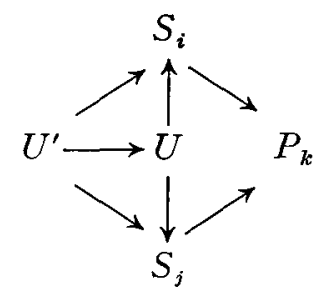

commutes. It follows that (5) is a pullback as required.

In case (ii) we have the commutative diagram

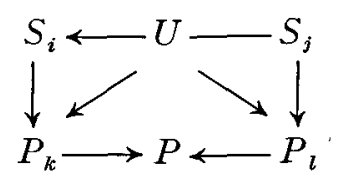

If, for some $U^{\prime}$ there exists $U^{\prime} \rightarrow S_{i}, U^{\prime} \rightarrow S_{j}$ such that

$$
U^{\prime} \rightarrow S_{i} \rightarrow P=U^{\prime} \rightarrow S_{j} \rightarrow P,
$$

then

$$
U^{\prime} \rightarrow S_{i} \rightarrow P_{k} \rightarrow P=U^{\prime} \rightarrow S_{j} \rightarrow P_{l} \rightarrow P .
$$

Thus there exist $U^{\prime} \rightarrow P_{k}\left(=U^{\prime} \rightarrow S_{i} \rightarrow P_{k}\right)$ and $U^{\prime} \rightarrow P_{l}\left(=U^{\prime} \rightarrow S_{j} \rightarrow P_{l}\right)$ such that

$$
U^{\prime} \rightarrow P_{k} \rightarrow P=U^{\prime} \rightarrow P_{l} \rightarrow P
$$

and so, by the pullback property of the diagram (4), there exists a unique $U^{\prime} \rightarrow U$ such that

$$
U^{\prime} \rightarrow P_{k}=U^{\prime} \rightarrow U \rightarrow P_{k}
$$

and

$$
U^{\prime} \rightarrow P_{l}=U^{\prime} \rightarrow U \rightarrow P_{l} .
$$

Thus, using (13) and the commutativity of (12), we have that

$$
U^{\prime} \rightarrow S_{i} \rightarrow P_{k}=U^{\prime} \rightarrow U \rightarrow P_{k}=U^{\prime} \rightarrow U \rightarrow S_{i} \rightarrow P_{k}
$$

and so

$$
U^{\prime} \rightarrow S_{i}=U^{\prime} \rightarrow U \rightarrow S_{i}
$$

since $S_{i} \rightarrow P_{k}$ is a monomorphism. Similarly, using (14), we find that

$$
U^{\prime} \rightarrow S_{j}=U^{\prime} \rightarrow U \rightarrow S_{j}
$$

and so (5) is a pullback as required, since the uniqueness of $U^{\prime} \rightarrow U$ in (15) and (16) follows from its uniqueness in (13) and (14). This completes the proof. 
Next we establish

THEOREM 1.4. If $U$ is a semigroup such that every amalgam $[\{S, T\}$; $U ;\{\varphi, \psi\}]$ of two semigroups with $C$ as core is embeddable, then every amalgam $\mathfrak{U}=\left[\left\{S_{\imath}: i \in I\right\} ; U ;\left\{\phi_{\imath}: i \in I\right\}\right]$ of arbitrarily many semigroups with $U$ as core is embeddable.

Proof. We apply Zorn's Lemma to the set $\mathscr{E}$ of subsets $E$ of $I$ such that the amalgam

$$
\mathfrak{H}_{E}=\left[\left\{S_{\imath}: i \in E\right\} ; U ;\left\{\varphi_{\imath}: i \in E\right\}\right]
$$

is embeddable. Then $\mathscr{E}$ contains all subsets of cardinal 2 and so is nonempty. Let $P_{E}$ denote the free product of the amalgam $\mathfrak{A}_{E}$ and let $\mathscr{C}$ be a chain in $\mathscr{E}$ with union $C$. If $E$ and $E^{\prime}$ in $\mathscr{C}$ are such that $E \subseteq E^{\prime}$ then

$$
P_{E^{\prime}} \cong P_{E}{ }_{U} P_{E^{\prime} \backslash E}
$$

by Theorem 1.3 and so there exists a natural monomorphism $P_{E} \rightarrow P_{E^{\prime}}$. Indeed $\left\{P_{E}: E \in \mathscr{C}\right\}$ with its associated monomorphisms forms a direct system with direct limit $L$. Now, if $i \in C$, then $i \in E$ for some $E$ in $\mathscr{C}$ and so there exists a monomorphism $S_{\imath} \rightarrow L$ which equals $S_{\imath} \rightarrow P_{E} \rightarrow L$ for every $E$ such that $i \in E$. Thus, for every $i, j$ in $C$ with $i \neq j$, we have a commutative diagram

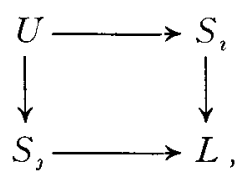

in which $S_{\imath} \rightarrow L$ and $S_{3} \rightarrow L$ are monomorphisms factoring through $P_{E}$, where $E$ is any member of $\mathscr{C}$ large enough to contain both $i$ and $j$. If there exist $U^{\prime}$ and homomorphisms $U^{\prime} \rightarrow S_{\imath}, U^{\prime} \rightarrow S_{3}$ such that

then

$$
U^{\prime} \rightarrow S_{\imath} \rightarrow L=U^{\prime} \rightarrow S_{\jmath} \rightarrow L,
$$

and so

$$
U^{\prime} \rightarrow S_{\imath} \rightarrow P_{E} \rightarrow L=U^{\prime} \rightarrow S_{\jmath} \rightarrow P_{E} \rightarrow L
$$

$$
U^{\prime} \rightarrow S_{\imath} \rightarrow P_{E}=U^{\prime} \rightarrow S_{\jmath} \rightarrow P_{E},
$$

since $P_{E} \rightarrow L$ is a monomorphism. Since the amalgam $\left[\left\{S_{2}, S_{\jmath}\right\} ; U ;\left\{\varphi_{2}, \varphi_{\vartheta}\right\}\right]$ is embedded in $P_{E}$, the diagram

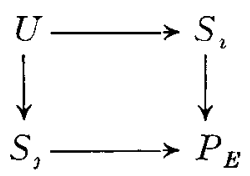

is a pullback for $S_{\imath} \rightarrow P_{E}, S_{\jmath} \rightarrow P_{E}$, and so there exists a unique $U^{\prime} \rightarrow U$ such that 


$$
U^{\prime} \rightarrow S_{i}=U^{\prime} \rightarrow U \rightarrow S_{i}, U^{\prime} \rightarrow S_{j}=U^{\prime} \rightarrow U \rightarrow S_{j} .
$$

Thus the diagram (17) is a pullback for $S_{i} \rightarrow L, S_{j} \rightarrow L$, and so the amalgam $\mathfrak{A}_{C}$ is embedded in $L$. [It can be shown that $L \cong P_{C}$, but we do not need this.] Thus $C \in \mathscr{E}$ and so by Zorn's Lemma $\mathscr{E}$ contains a maximal element $J$. If $J \neq I$, let $K=J \cup\{i\}$, where $i \notin J$. Then $\mathfrak{A}_{K}$ is embeddable by Theorem 1.3 and by the assumed embeddability of amalgams of two semigroups with $U$ as core, and so the maximality of $J$ is contradicted. Thus $J=I$ and so $\mathfrak{A}$ is embeddable as required.

\section{Examples}

The first example shows that an amalgam $[\{S, T\} ; U ;\{\varphi, \psi\}]$ need not be embeddable even if $\varphi(U)$ and $\psi(U)$ are self-dominating in $S$ and $T$ respectively.

Let $U$ be the quotient of the free commutative semigroup $F_{U}$ on four generators $u_{1}, u_{2}, u_{3}, u_{4}$ by the congruence $\alpha$ generated by

$$
\mathfrak{A}=\left\{\left(u_{1} u_{3}, u_{2} u_{4}\right),\left(u_{1} u_{4}, u_{2} u_{3}\right),\left(u_{1}^{2}, u_{2}^{2}\right),\left(u_{3}^{2}, u_{4}^{2}\right)\right\} .
$$

Let $S$ be the quotient of the free commutative semigroup $F_{S}$ on six generators $v_{1}, v_{2}, v_{3}, v_{4}, s_{1}, s_{2}$ by the congruence $\beta$ generated by

$$
\mathfrak{B}=\left\{\left(v_{1} v_{3}, v_{2} v_{4}\right),\left(v_{1} v_{4}, v_{2} v_{3}\right),\left(v_{1}^{2}, v_{2}^{2}\right),\left(v_{3}^{2}, v_{4}^{2}\right),\left(s_{1} v_{2}, s_{2} v_{3}\right)\right\} .
$$

Let $T$ be the quotient of the free commutative semigroup $F_{T}$ on five generators $w_{1}, w_{2}, w_{3}, w_{4}, t$ by the congruence $\gamma$ generated by

$$
\mathfrak{C}=\left\{\left(w_{1}, w_{2} t\right),\left(w_{2}, w_{1} t\right),\left(w_{3}, w_{4} t\right),\left(w_{4}, w_{3} t\right)\right\} .
$$

It is convenient to summarise the relevant facts about these semigroups in the following theorem.

ThEOREM 2.1. (i) The mapping $\varphi: U \rightarrow S$ defined by

is a monomorphism.

$$
\varphi\left(u_{i} \alpha\right)=v_{i} \beta \quad(i=1,2,3,4)
$$

(ii) $\varphi(U)$ is self-dominating in $S$.

(iii) $\left(s_{1} v_{1}\right) \beta \neq\left(s_{2} v_{4}\right) \beta$.

(iv) The mapping $\psi: U \rightarrow T$ defined by

is a monomorphism.

$$
\psi\left(u_{i} \alpha\right)=w_{i} \gamma \quad(i=1,2,3,4)
$$

(v) $\psi(U)$ is self-dominating in $T$.

(vi) If $\mu$ is the canonical mapping of $S$ into the free product $P$ of the amalgam $[\{S, T\} ; U ;\{\varphi, \psi\}]$, then

$$
\mu\left(\left(s_{1} v_{1}\right) \beta\right)=\mu\left(\left(s_{2} v_{4}\right) \beta\right) .
$$


Before proving these assertions, let us note that it follows from (iii) and (vi) that the amalgam $[\{S, T\} ; U ;\{\varphi, \psi\}]$ is not embeddable.

Proof of Theorem 2.1. (i) It is clear from the definitions of $\mathfrak{A}$ and $\mathfrak{B}$ that $\varphi$ is well-defined. In fact it is also one-one; for if

$$
\varphi\left(\left(u_{1}^{m_{1}} u_{2}^{m_{2}} u_{3}^{m_{3}} u_{4}^{m_{4}}\right) \alpha\right)=\varphi\left(\left(u_{1}^{n_{1}} u_{2}^{n_{2}} u_{3}^{n_{3}} u_{4}^{n_{4}}\right) \alpha\right),
$$

then $v_{1}^{m_{1}} v_{2}^{m_{2}} v_{3}^{m_{3}} v_{4}^{m_{4}}$ and $v_{1}^{n_{1}} v_{2}^{n_{2}} v_{3}^{n_{3}} v_{4}^{n_{4}}$ are connected by a sequence of elementary $\mathfrak{B}$-transitions. Now clearly the last relation $\left(s_{1} v_{2}, s_{2} v_{3}\right)$ in $\mathfrak{B}$ can never be used in this sequence; hence $u_{1}^{m_{1}} u_{2}^{m_{2}} u_{3}^{m_{3}} u_{4}^{m_{4}}$ and $u_{1}^{n_{1}} u_{2}^{n_{2}} u_{3}^{n_{3}} u_{4}^{n_{4}}$ are connected in an obvious way by a sequence of elementary $\mathfrak{A}$-transitions - which is what we require.

(ii) Consider the two homomorphisms $\chi$ and $\omega$ of $S$ into the infinite monogenic semigroup $M=\left\{1, x, x^{2}, \cdots\right\}$, defined as follows:

$$
\begin{gathered}
\chi(s)=1 \text { for every } s \text { in } S ; \\
\omega\left(v_{i} \beta\right)=1(i=1,2,3,4) ; \omega\left(s_{j} \beta\right)=x(j=1,2) .
\end{gathered}
$$

Then clearly $\chi(s)=\omega(s)$ if and only if $s \in \varphi(U)$ and so $\varphi(U)$ is its own dominion in $S$.

(iii) By inspection, no elementary $\mathfrak{B}$-transition can change the word $s_{1} v_{1}$ into any other word. This is also the case for $s_{2} v_{4}$ and so certainly $\left(s_{1} v_{1}, s_{2} v_{4}\right) \notin \beta$.

(iv) This is less trivial. First notice that $\mathfrak{D} \cong \gamma$, where

$$
\mathfrak{D}=\left\{\left(w_{1} w_{3}, w_{2} w_{4}\right),\left(w_{1} w_{4}, w_{2} w_{3}\right),\left(w_{1}^{2}, w_{2}^{2}\right),\left(w_{3}^{2}, w_{4}^{2}\right)\right\} ;
$$

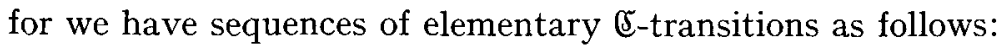

$$
\begin{aligned}
w_{1} w_{3} & \rightarrow w_{2} w_{3} t \rightarrow w_{2} w_{4}, w_{1} w_{4} \rightarrow w_{2} w_{4} t \rightarrow w_{2} w_{3}, \\
w_{1}^{2} & \rightarrow w_{1} w_{2} t \rightarrow w_{2}^{2}, \quad w_{3}^{2} \rightarrow w_{3} w_{4} t \rightarrow w_{4}^{2} .
\end{aligned}
$$

It follows that $\psi$ is well-defined, but it is not immediately clear that $\psi$ is one-one. We must show that if two words in $w_{1}, w_{2}, w_{3}, w_{4}$ are connected by a sequence of elementary $\mathfrak{C}$-transitions, then they are connected by a sequence of elementary $D$-transitions; for it then follows that the corresponding two words in $u_{1}, u_{2}, u_{3}, u_{4}$ are connected by a sequence of elementary $\mathfrak{A}$-transitions.

It is convenient to begin by considering a sequence $\mathscr{S}$ of elementary $\mathfrak{E}$-transitions beginning on a general element $x$ of $F_{T}$. The element $x$ is a word of the form

$$
w_{1}^{m_{1}} w_{2}^{m_{2}} w_{3}^{m_{3}} w_{4}^{m_{4}} t^{m}
$$

and so can conveniently be identified with the quintuple

$$
\left(m_{1}, m_{2}, m_{3}, m_{4}, m\right)
$$


of non-negative integers. Let us now name the ordered pairs constituting ce as follows:

$$
\begin{aligned}
& \mathfrak{c}_{1}=\left(w_{1}, w_{2} t\right), \wedge \quad\left(w_{2}, w_{1} t\right) \\
& \mathfrak{c}_{3}=\left(w_{3}, w_{4} t\right), c_{4}=\left(w_{4}, w_{3} t\right) .
\end{aligned}
$$

In the sequence $\mathscr{S}$, an elementary $\mathfrak{C}$-transition will be called a forward step if it increases the number of occurrences of $t$ by one, and a backward step if it decreases the number of occurrences of $t$ by one. The weight of $\mathfrak{c}_{i}(i=1,2,3,4)$ in the sequence $\mathscr{S}$ is defined to be the number of forward steps based on $\mathfrak{c}_{i}$ minus the number of backward steps based on $\mathfrak{c}_{i}$. The weight of the sequence $\mathscr{S}$ is defined to be the quadruple $\left(r_{1}, r_{2}, r_{3}, r_{4}\right)$ of integers (positive, negative or zero), where $r_{i}(i=1,2,3,4)$ is the weight of $\mathfrak{c}_{i}$ in $\mathscr{S}$.

By a straightforward inductive argument one can prove

Lemma 2.2. If $\mathscr{S}$ is a sequence of reight $\left(r_{1}, r_{2}, r_{3}, r_{4}\right)$ of elementary (C-transitions beginning on the element $\left(m_{1}, m_{2}, m_{3}, m_{4}, m\right)$ of $F_{r}$, then $\mathscr{S}$ ends on the element

$$
\left(m_{1}-r_{1}+r_{2}, m_{2}+r_{1}-r_{2}, m_{3}-r_{3}+r_{4}, m_{4}+r_{3}-r_{4}, m+r_{1}+r_{2}+r_{3}+r_{4}\right) .
$$

Our interest is of course primarily in sequences that connect two elements $\left(m_{1}, m_{2}, m_{3}, m_{4}, 0\right)$ and $\left(n_{1}, n_{2}, n_{3}, n_{4}, 0\right)$. If such a sequence has weight $\left(r_{1}, r_{2}, r_{3}, r_{4}\right)$, then $r_{1}+r_{2}+r_{3}+r_{4}=0$.

The next lemma contains the result we require.

Lemma 2.3. If $\left(m_{1}, m_{2}, m_{3}, m_{4}, 0\right)$ and $\left(n_{1}, n_{2}, n_{3}, n_{4}, 0\right)$ are $\gamma$-equivalent, then there exists a sequence of elementary $D$-transitions connecting them.

Proof. In describing sequences of elementary $D$-transitions, I shall use phrases such as " $k$ uses of $w_{1}^{2} \rightarrow w_{2}^{2}$ ". If $k$ is non-negative the meaning of this is clear; if $k$ is negative, the phrase is to be interpreted as meaning " $-k$ uses of $w_{2}^{2} \rightarrow w_{1}^{2 " .}$.

By hypothesis, there exists a sequence of weight $\left(r_{1}, r_{2}, r_{3}, r_{4}\right)$ of elementary (5-transitions connecting $\left(m_{1}, m_{2}, m_{3}, m_{4}, 0\right)$ to $\left(n_{1}, n_{2}, n_{3}, n_{4}, 0\right)$, and, as we have seen, $r_{1}+r_{2}+r_{3}+r_{4}=0$. Notice that $r_{1}-r_{2}$ is even if and only if $r_{1}+r_{2}$ is even; this in turn holds if and only if $r_{3}+r_{4}$ is even, that is, if and only if $r_{3}-r_{4}$ is even.

By Lemma 2.2,

$$
\left(n_{1}, n_{2}, n_{3}, n_{4}, 0\right)=\left(m_{1}-r_{1}+r_{2}, m_{2}+r_{1}-r_{2}, m_{3}-r_{3}+r_{4}, m_{4}+r_{3}-r_{4}, 0\right) .
$$

If $r_{1}-r_{2}$ and $r_{3}-r_{4}$ are both even, then a sequence consisting of $\frac{1}{2}\left(r_{1}-r_{2}\right)$ uses of $w_{1}^{2} \rightarrow w_{2}^{2}$ followed by $\frac{1}{2}\left(r_{3}-r_{4}\right)$ uses of $w_{3}^{2} \rightarrow w_{4}^{2}$ will change $\left(m_{1}, m_{2}, m_{3}, m_{4}, 0\right)$ to $\left(n_{1}, n_{2}, n_{3}, n_{4}, 0\right)$. A typical intermediate element 
in the sequence will be $\left(k_{1}, k_{2}, k_{3}, k_{4}, 0\right)$, where $k_{i}$ lies between $m_{i}$ and $n_{i}$ for each $i$, and so negative values never appear.

Now suppose that $r_{1}-r_{2}=2 k+1, r_{3}-r_{4}=2 l+1$, where $k$ and $l$ are integers. Thus

$$
\begin{aligned}
& n_{1}=m_{1}-2 k-1, \quad n_{2}=m_{2}+2 k+1, \\
& n_{3}=m_{3}-2 l-1, \quad n_{4}=m_{4}+2 l+1 .
\end{aligned}
$$

If we apply a sequence of elementary $\mathfrak{D}$-transitions consisting of $k$ uses of $w_{1}^{2} \rightarrow w_{2}^{2}$ followed by $l$ uses of $w_{3}^{2} \rightarrow w_{4}^{2}$, then we change $\left(m_{1}, m_{2}, m_{3}, m_{4}, 0\right)$ to

$$
\left(m_{1}-2 k, m_{2}+2 k, m_{3}-2 l, m_{4}+2 l, 0\right) .
$$

This is legitimate unless $n_{2}=0$ or $n_{4}=0$, in which case we should have $m_{2}+2 k=-1$ or $m_{4}+2 l=-1$. Suppose for the moment that $n_{2}>0$ and $n_{4}>0$. Then a single use of $w_{1} w_{3} \rightarrow w_{2} w_{4}$ transforms the quintuple (18) into $\left(n_{1}, n_{2}, n_{3}, n_{4}, 0\right)$ as required.

If $n_{2}=0$ (and $n_{4}>0$ ), then $m_{2}+2 k=-1$. Since $m_{2}>0$ we must therefore have that $k \leqq-1$; hence $m_{1}-2 k-2 \geqq m_{1} \geqq 0$. Now, $k+1$ uses of $\varkappa_{1}^{2} \rightarrow w_{2}^{2}$ followed by $l$ uses of $w_{3}^{2} \rightarrow w_{4}^{2}$ will change $\left(m_{1}, m_{2}, m_{3}, m_{4}, 0\right)$ to

$$
\left(m_{1}-2 k-2, m_{2}+2 k+2, m_{3}-2 l, m_{4}+2 l, 0\right),
$$

and a single use of $w_{2} w_{3} \rightarrow w_{1} w_{4}$ now changes this to $\left(n_{1}, n_{2}, n_{3}, n_{4}, 0\right)$ as required. Similar modifications are effective if $n_{2}>0$ and $n_{4}=0$ or if $n_{2}=n_{4}=0$.

This completes the proof that $\psi$ is a monomorphism.

(v) It is clear that $\psi(U)$ is an ideal in $T$. If, therefore, we take $\chi$ as the natural homomorphism of $T$ onto the Rees factor semigroup $T / \psi(U)$ and $\omega$ as the homomorphism mapping every element of $T$ to the zero of $T / \psi(U)$, then obviously $\chi(x)=\omega(x) \quad(x \in T)$ if and only if $x \in \psi(U)$. Thus $\psi(U)$ is its own dominion in $T$.

(vi) The free product $P$ of the amalgam $[\{S, T\} ; U ;\{\varphi, \psi\}]$ can be described as the quotient of the free commutative semigroup on the seven generators $u_{1}, u_{2}, u_{3}, u_{4}, s_{1}, s_{2}, t$ by the congruence $\pi$ generated by

\section{Hence}

$$
\left.\mathfrak{P}=\left\{s_{1} u_{2}, s_{2} u_{3}\right),\left(u_{1}, u_{2} t\right),\left(u_{2}, u_{1} t\right),\left(u_{3}, u_{4} t\right),\left(u_{4}, u_{3} t\right)\right\} .
$$

$$
\begin{aligned}
\mu\left(\left(s_{1} v_{1}\right) \beta\right) & =\left(s_{1} u_{1}\right) \pi=\left(s_{1} u_{2} t\right) \pi=\left(s_{2} u_{3} t\right) \pi \\
& =\left(s_{2} u_{4}\right) \pi=\mu\left(\left(s_{2} v_{4}\right) \beta\right) .
\end{aligned}
$$

This completes the proof.

The next example is of an amalgam $[\{S, T\} ; U ;\{\varphi, \psi\}]$ which is embeddable, but such that $\varphi(U)$ is not self-dominating in $S$ and $\psi(U)$ is not self-dominating in $T$. 
Let $U$ be the free commutative semigroup generated by $u_{1}, \cdots, u_{6}$. Let $S$ be the quotient of the free commutative semigroup $F_{S}$ on eight generators $v_{1}, \cdots, v_{6}, x, y$ by the congruence $\alpha$ generated by

$$
\mathfrak{A}=\left\{\left(v_{1}, x v_{2}\right),\left(v_{3}, v_{2} y\right)\right\} .
$$

Let $T$ be the quotient of the free commutative semigroup $F_{T}$ on eight generators $w_{1}, \cdots, w_{6}, z, t$ by the congruence $\beta$ generated by

$$
\mathfrak{B}=\left\{\left(w_{4}, z w_{5}\right),\left(w_{6}, w_{5} t\right)\right\} .
$$

Again we summarise the relevant facts in a theorem.

Theorem 2.4. (i) The mapping $\varphi: U \rightarrow S$ defined by

$$
\varphi\left(u_{i}\right)=v_{i} \alpha \quad(i=1, \cdots, 6)
$$

is a monomorphism.

(ii) The mapping $\psi: U \rightarrow T$ defined by

is a monomorphism.

$$
\psi\left(u_{i}\right)=w_{i} \beta
$$$$
(i=1, \cdots, 6)
$$

(iii) $\varphi(U)$ is not self-dominating in $S$.

(iv) $\psi(U)$ is not self-dominating in $T$.

(v) The amalgam $[\{S, T\} ; U ;\{\varphi, \psi\}]$ is embeddable.

Proof. By the symmetry of the situation, it will clearly suffice to prove (i), (iii) and (v).

(i) A typical element of $F_{S}$ is

$$
v_{1}^{n_{1}} \cdots v_{6}^{n_{6}} x^{p} y^{q},
$$

which it is convenient to identity with the octuple $\left(n_{1}, \cdots, n_{6}, p, q\right)$ of nonnegative integers. If $\left(n_{1}, \cdots, n_{6}, 0,0\right)$ is an arbitrary element of the subsemigroup $V$ of $F_{S}$ generated by $v_{1}, \cdots, v_{6}$, then any element of $F_{S}$ derived from this by a sequence of elementary $\mathfrak{U}$-transitions is necessarily of the form

$$
\left(n_{1}-r, n_{2}+r+s, n_{3}-s, n_{4}, n_{5}, n_{6}, r, s\right)
$$

where $r, s$ are non-negative integers. This element belongs to $V$ only if $r=s=0$ and so $\alpha \cap(V \times V)$ is the identical equivalence on $V$ - which is exactly what we require in order to show that $\varphi$ is a monomorphism.

(iii) It follows from the considerations above that an element $\left(n_{1}, \cdots, n_{6}, p, q\right) \alpha$ of $S$ belongs to $\varphi(U)$ if and only if $n_{2}-p-q \geqq 0$. This condition is certainly not satisfied by

$$
\left(v_{1} y\right) \alpha=(1,0,0,0,0,0,0,1) \alpha
$$

and so $\left(v_{1} y\right) \alpha \notin \varphi(U)$. However, if $\gamma$ and $\delta$ are two homomorphisms of $S$ 
which coincide on $\varphi(U)$, then, denoting $f \propto$ by $f$ for any element $f$ in $F_{S}$, we find that

$$
\begin{aligned}
\gamma\left(\boldsymbol{v}_{\mathbf{1}} \boldsymbol{y}\right) & =\gamma\left(\boldsymbol{v}_{\mathbf{1}}\right) \gamma(\boldsymbol{y})=\delta\left(\boldsymbol{v}_{\mathbf{1}}\right) \gamma(\boldsymbol{y}) \\
& =\delta\left(\boldsymbol{x} \boldsymbol{v}_{\mathbf{2}}\right) \gamma(\boldsymbol{y})=\delta(\boldsymbol{x}) \delta\left(\boldsymbol{v}_{\mathbf{2}}\right) \gamma(\boldsymbol{y})=\delta(\boldsymbol{x}) \gamma\left(\boldsymbol{v}_{\mathbf{2}}\right) \gamma(\boldsymbol{y}) \\
& =\delta(\boldsymbol{x}) \gamma\left(\boldsymbol{v}_{\mathbf{2}} \boldsymbol{y}\right)=\delta(\boldsymbol{x}) \gamma\left(\boldsymbol{v}_{3}\right)=\delta(\boldsymbol{x}) \delta\left(\boldsymbol{v}_{\mathbf{3}}\right) \\
& =\delta\left(\boldsymbol{x} \boldsymbol{v}_{\mathbf{3}}\right)=\delta\left(\boldsymbol{x} \boldsymbol{v}_{2} \boldsymbol{y}\right)=\delta\left(\boldsymbol{v}_{\mathbf{1}} \boldsymbol{y}\right)
\end{aligned}
$$

Thus $\boldsymbol{V}_{\mathbf{1}} \boldsymbol{y}$ is in the dominion of $\varphi(U)$ in $S$ and so $\varphi(U)$ is not self-dominating.

(v) The free product $P$ of the amalgam $[\{S, T\} ; U ;\{\varphi, \psi\}]$ can be described as the quotient of the free commutative semigroup $F_{P}$ on the ten generators $u_{1}, \cdots, u_{6}, x, y, z, t$ by the congruence $\pi$ generated by

$$
\mathfrak{P}=\left\{\left(u_{1}, x u_{2}\right),\left(u_{3}, u_{2} y\right),\left(u_{4}, z u_{5}\right),\left(u_{6}, u_{5} t\right)\right\},
$$

the canonical homomorphisms $\mu: S \rightarrow P, v: T \rightarrow P$ being given by

$$
\begin{aligned}
& \mu\left(v_{i} \alpha\right)=u_{i} \pi(i=1, \cdots, 6), \quad \mu(x \alpha)=x \pi, \quad \mu(y \alpha)=y \pi \\
& \nu\left(w_{i} \beta\right)=u_{i} \pi(i=1, \cdots, 6), \quad \nu(z \beta)=z \pi, \quad v(t \beta)=t \pi .
\end{aligned}
$$

The elements $u_{1}^{n_{1}} \cdots u_{\mathbf{6}}^{n_{6}} x^{j} y^{k} z^{l} t^{m}$ of $F_{P}$ can conveniently be identified with 10-tuples

$$
\left(n_{1}, \cdots, n_{6}, j, k, l, m\right)
$$

of non-negative integers. Thus, if

$$
s=\left(n_{1}, \cdots, n_{6}, j, k\right) \alpha, \quad s^{\prime}=\left(n_{1}^{\prime}, \cdots, n_{6}^{\prime}, j^{\prime}, k^{\prime}\right) \alpha
$$

are two elements of $S$, then the supposition that $\mu(s)=\mu\left(s^{\prime}\right)$ amounts to the supposition that the two 10-tuples

$$
\left(n_{1}, \cdots, n_{6}, j, k, 0,0\right), \quad\left(n_{1}^{\prime}, \cdots, n_{6}^{\prime}, j^{\prime}, k^{\prime}, 0,0\right)
$$

in $F_{P}$ are connected by a sequence of elementary $\mathfrak{P}$-transitions. It is easy to see that the most general element of $F_{\boldsymbol{P}}$ obtainable from $\left(n_{1}, \cdots, n_{\mathbf{6}}, j, k, 0,0\right)$ by elementary $\mathfrak{\$}$-transitions is

$$
\left(n_{1}-p, n_{2}+p+q, n_{3}-q, n_{4}-r, n_{5}+r+s, n_{6}-s, j+p, k+q, r, s\right),
$$

where $r$ and $s$ are non-negative integers and $p$ and $q$ are integers such that $j+p, k+q$ are non-negative. Putting $r=s=0$, we find that

$$
s^{\prime}=\left(n_{1}-p, n_{2}+p+q, n_{3}-q, n_{4}, n_{5}, n_{6}, j+p, k+q\right) \alpha .
$$

Hence $s=s^{\prime}$, since it is clear that the two octuples

$$
\left(n_{1}, \cdots, n_{6}, j, k\right),\left(n_{1}-p, n_{2}+p+q, n_{3}-q, n_{4}, n_{5}, n_{6}, j+p, k+q\right)
$$

can be connected by a sequence of elementary $\mathfrak{A}$-transitions. A similar argument establishes that $\boldsymbol{v}$ is a monomorphism. 
Suppose now that

$$
t=\left(n_{1}^{\prime}, \cdots, n_{6}^{\prime}, l, m\right) \beta
$$

is an element of $T$ and that $\mu(s)=v(t)$, where $s$ is as before. Then there exists a sequence of elementary $\mathfrak{B}$-transitions connecting

$$
\left(n_{1}, \cdots, n_{6}, j, k, 0,0\right) \text { and }\left(n_{1}^{\prime}, \cdots, n_{6}^{\prime}, 0,0, l, m\right) \text {. }
$$

It follows from (19) that $p=-j, q=-k$ and so

$$
n_{1}^{\prime}=n_{1}+j, n_{2}^{\prime}=n_{2}-j-k, n_{3}^{\prime}=n_{3}+k .
$$

It is now easy to see that the element $\left(n_{1}, \cdots, n_{6}, j, k\right)$ of $F_{S}$ can be connected to the element $\left(n_{1}^{\prime}, n_{2}^{\prime}, n_{3}^{\prime}, n_{4}, n_{5}, n_{6}, 0,0\right)$ by means of a sequence of elementary $\mathfrak{A}$-transitions, and so $s \in \varphi(U)$ as required.

This completes the proof.

\section{3. $C S g$-amalgamable semigroups}

By virtue of Theorem 1.4 we can restrict our attention to amalgams of two semigroups. Accordingly, let $\mathfrak{A}=[\{S, T\} ; U ;\{\varphi, \psi\}]$ be a commutative semigroup amalgam. It will be convenient to denote $\varphi(U)$ and $\psi(U)$ by $V$ and $W$ respectively and to write $v, v_{i}, v^{\prime}$, etc. and $w, w_{i}$, $w^{\prime}$, etc. respectively for $\varphi(u), \varphi\left(u_{i}\right), \varphi\left(u^{\prime}\right)$, etc. and $\psi(u), \psi\left(u_{i}\right), \psi\left(u^{\prime}\right)$, etc.

The coproduct [6] of $S$ and $T$ in the category $C S g$ is most easily described as follows: first form $S^{(1)}$ and $T^{(1)}$ by adjoining an extra unity element 1 to each of $S$ and $T$ whether or not they already have unities; then form the Cartesian product $S^{(1)} \times T^{(1)}$; then remove the element $(1,1)$. Since this semigroup is the commutative analogue of the free product of $S$ and $T$ as usually understood in semigroup theory, it is reasonable to write it as $S * T$. It contains isomorphic copies $\{(s, 1): s \in S\},\{(1, t): t \in T\}$ of $S$ and $T$ respectively.

The free product $P=S *{ }_{U} T$ of the amalgam $\mathfrak{A}$ is thus given by

$$
P=(S * T) / \rho,
$$

where $\rho$ is the congruence on $S * T$ generated by

$$
\Re=\{((\varphi(u), 1),(1, \psi(u))): u \in U\},
$$

and the canonical homomorphisms $\mu: S \rightarrow P, v: T \rightarrow P$ are given by

$$
\mu(s)=(s, 1) \rho, v(t)=(1, t) \rho .
$$

We first obtain general necessary and sufficient conditions for the embeddability of the amalgam $\mathfrak{A}$, analogous to the commutative zigzag theorem (1.1) in [4]. 
Suppose that $(x, y), \quad(z, t) \in S * T$ and that $(x, y) \rightarrow(z, t)$ by an elementary $\Re$-transition. Then either

or

$$
(x, y)=(p, q)(v, 1)(r, s),(z, t)=(p, q)(1, w)(r, s),
$$

$$
(x, y)=(p, q)(1, w)(r, s),(z, t)=(p, q)(v, \mathbf{1})(r, s),
$$

where $p, r \in S^{(1)}, q, s \in T^{(1)}, v=\varphi(u), w=\psi(u), u \in U$. A step of the first type will be called an $r$-step (since the $v$ moves right); one of the second type will be called an $l$-step. By commutativity we thus have either

or

$$
x=z v, \quad t=w y,
$$

$$
z=x v, \quad y=w t .
$$

Two consecutive $r$-steps (corresponding to $u_{1}, u_{2}$ respectively) can be combined into a single $r$-step (corresponding to $u_{2} u_{1}$ ). A similar remark applies to $l$-steps and hence we may assume that every sequence of elementary $\Re$-transitions consists of $l$-steps and $r$-steps alternately.

If $\mu(s)=\mu\left(s^{\prime}\right)$, where $s, s^{\prime} \in S$, then there exists a sequence

$$
(s, 1) \rightarrow \cdots \rightarrow\left(s^{\prime}, 1\right)
$$

of elementary $\Re$-transitions. The first step must be an $r$-step, since $I$ has no divisors in $S$; similarly the last step must be an $l$-step. Hence there exist $s_{1}, \cdots, s_{n} \in S, t_{1}, \cdots, t_{n-1} \in T, u_{1}, \cdots, u_{2 n} \in U$ such that

$$
\begin{aligned}
s & =s_{1} v_{1}, \quad w_{1}=w_{2} t_{1}, \\
s_{1} v_{2} & =s_{2} v_{3}, \quad w_{3} t_{1}=w_{4} t_{2}, \\
\cdots & \\
s_{n-1} v_{2 n-2} & =s_{n} v_{2 n-1}, \quad w_{2 n-1} t_{n-1}=w_{2 n}, \\
s_{n} v_{2 n} & =s^{\prime} .
\end{aligned}
$$

Thus $\mu$ is one-one if and only if the equalities

$$
\begin{gathered}
w_{1}=w_{2} t_{1}, \\
s_{1} v_{2}=s_{2} v_{3}, \quad w_{3} t_{1}=w_{4} t_{2}, \\
\ldots \quad \\
s_{n-1} v_{2 n-2}=s_{n} v_{2 n-1}, \quad w_{2 n-1} t_{n-1}=w_{2 n}
\end{gathered}
$$

imply that $s_{1} v_{1}=s_{n} v_{2 n}$.

A similar argument shows that $v$ is one-one if and only if the equalities 
(21)

$$
\begin{aligned}
v_{1} & =s_{1} v_{2}, \quad w_{2} t_{1}=w_{3} t_{2}, \\
s_{1} v_{3} & =s_{2} v_{4}, \quad w_{4} t_{2}=w_{5} t_{3}, \\
& \ldots \\
s_{n-2} v_{2 n-3} & =s_{n-1} v_{2 n-2}, \quad w_{2 n-2} t_{n-1}=w_{2 n-1} t_{n}, \\
s_{n-1} v_{2 n-1} & =v_{2 n}
\end{aligned}
$$

(where $s_{1}, \cdots, s_{n-1} \in S, t_{1}, \cdots, t_{n} \in T, u_{1}, \cdots, u_{2 n} \in U$ ) imply that $w_{1} t_{1}=$ $w_{2 n} t_{n}$.

Also, if $\mu$ and $\nu$ are one-one, then $\mu(S) \cap \nu(T)=\mu \varphi(U)(=\nu \psi(U))$ if and only if the equalities

$$
\begin{aligned}
& w_{1}=w_{2} t_{1}, \\
& s_{1} v_{2}=s_{2} v_{3}, \quad w_{3} t_{1}=w_{4} t_{2}, \\
& \cdots \\
& s_{n-2} v_{2 n-4}=s_{n-1} v_{2 n-3}, \quad w_{2 n-3} t_{n-2}=w_{2 n-2} t_{n-1}, \\
& s_{n-1} v_{2 n-2}=v_{2 n-1}
\end{aligned}
$$

(where $s_{1}, \cdots, s_{n-1} \in S, t_{1}, \cdots, t_{n-1} \in T, u_{1}, \cdots, u_{2 n-1} \in U$ ) imply that $s_{1} v_{1} \in V$.

THEOREM 3.1. If $\mathfrak{A}=[\{S, T\} ; U ;\{\varphi, \psi\}]$ is a' commutative semigroup amalgam and $U$ is regular, then $\mathscr{A}$ is embeddable.

Proof. Since $U$ is both commutative and regular, it is an inverse semigroup; that is, every element $x$ of $U$ has a unique inverse $x^{-1}$ such that

$$
x x^{-1} x=x, \quad x^{-1} x x^{-1}=x^{-1} .
$$

Suppose now that we have a set of equalities (20). Let $w_{r} w_{r}^{-1}=w_{r}^{-1} w_{m}=e_{r}$ for $r=1, \cdots, 2 n$, and let $f_{r}=e_{2} e_{4} \cdots e_{2 r}(r=1, \cdots, n)$, Then

Lemma 3.2. If $t_{r}^{*}=f_{r} t_{r}(r=1, \cdots, n-1)$, then $t_{r}^{*} \in W$, and

$$
w_{1}=w_{2} t_{1}^{*}, \quad w_{2 r-1} t_{r-1}^{*}=w_{2 r} t_{r}^{*} \quad(r=2, \cdots, n-1),
$$

PROOF. First,

$$
t_{1}^{*}=f_{1} t_{1}=e_{2} t_{1}=w_{2}^{-1} w_{2} t_{1}=w_{2}^{-1} w_{1} \in W .
$$

Also, if $t_{r-1}^{*} \in W$, then

$$
\begin{aligned}
t_{r}^{*} & =f_{r} t_{r}=f_{r-1} e_{2 r} t_{r}=f_{r-1} w_{2 r}^{-1} w_{2 r} t_{r} \\
& =f_{r-1} w_{2 r}^{-1} w_{2 r-1} t_{r-1} \quad(\text { by }(20)) \\
& =w_{2 r}^{-1} w_{2 r-1} t_{r-1}^{*} ;
\end{aligned}
$$


hence $t_{r}^{*} \in W$. Thus the first statement in the lemma is proved by induction.

Also,

$$
\begin{aligned}
w_{1}= & w_{2} t_{1}=w_{2} e_{2} t_{1}=w_{2} t_{1} t_{1}=w_{2} t_{1}^{*}, \\
w_{2 r-1} t_{r-1}^{*}= & f_{r-1} w_{2 r-1} t_{r-1}=f_{r-1} w_{2 r} t_{r} \\
= & f_{r-1} e_{2 r} w_{2 r} t_{r}=f_{r} w_{2 r} t_{r}=w_{2 r} t_{r}^{*} \\
& (r=2, \cdots, n-1), \\
w_{2 n-1} t_{n-1}^{*}= & f_{n-1} w_{2 n-1} t_{n-1}=f_{n-1} w_{2 n},
\end{aligned}
$$

and so the lemma is proved.

Returning now to the proof of Theorem 3.1, if we let $s_{r}^{*}=\varphi \psi^{-1}\left(t_{r}^{*}\right)$ $(r=1, \cdots, n-1)$, we have that

$$
\begin{aligned}
s_{1} v_{1} & =s_{1} v_{2} s_{1}^{*}=s_{2} v_{3} s_{1}^{*}=s_{2} v_{4} s_{2}^{*} \\
& =\cdots=s_{n-1} v_{2 n-2} s_{n-1}^{*}=s_{n} v_{2 n-1} s_{n-1}^{*}=s_{n} e v_{2 n} \\
& =e s_{n} v_{2 n},
\end{aligned}
$$

where $e=\varphi \psi^{-1}\left(f_{n-1}\right)$ is an idempotent of $V$. However, the situation is symmetrical and so we can equally assert that $s_{n} v_{2 n}=f s_{1} v_{1}$, where $f$ is an idempotent of $V$. Hence

$$
\begin{aligned}
s_{1} v_{1} & =e s_{n} v_{2 n}=e f s_{n} v_{2 n}\left(\text { since } f s_{n} v_{2 n}=s_{n} v_{2 n}\right) \\
& =f e s_{n} v_{2 n}=f s_{1} v_{1}=s_{n} v_{2 n},
\end{aligned}
$$

and so $\mu: S \rightarrow P$ is a monomorphism.

The proof that $v: T \rightarrow P$ is a monomorphism is similar.

Finally, suppose that we have equalities (22), and take $t_{1}^{*}, \cdots, t_{n-1}^{*}$ as before. Then the result of Lemma 3.2 holds, and if we again write $s_{r}^{*}$ for $\varphi \psi^{-1}\left(t_{r}^{*}\right)$, we obtain

$$
s_{1} v_{1}=s_{1} v_{2} s_{1}^{*}=\cdots=s_{n-1} v_{2 n-2} s_{n-1}^{*}=v_{2 n-1} s_{n-1}^{*} \in V .
$$

This completes the proof.

Before stating the next theorem, we recall some definitions in [4]. First, if $c, d$ are distinct elements of a commutative semigroup $S$, we say that $c$ is a potential divisor of $d$ if, for every $a, b \in S^{1}$,

$$
a c=b c \Rightarrow a d=b d .
$$

$S$ is called division-ordered if every potential divisor is an actual divisor. If $S$ is division-ordered and if, for any two distinct elements $x, y$ of $S$, either $x$ divides $y$ or $y$ divides $x$, we say that $S$ is totally division-ordered.

It is known ([4], Theorem 2.6) that totally division ordered commutative semigroups are $\boldsymbol{C S g}$-closed. In fact we even have 
THEOREM 3.3. If $\mathfrak{A}=[\{S, T\} ; U ;\{\varphi, \psi\}]$ is a commutative semigroup amalgam and $U$ is totally division-ordered, then $\mathfrak{A}$ is embeddable.

Proof. With the same notation as before, suppose that $\mu: S \rightarrow P$ is not a monomorphism. Then there exist $s, s^{\prime} \in S$ such that $\mu(s)=\mu\left(s^{\prime}\right)$, but $s \neq s^{\prime}$. That is, there exist $u_{1}, \cdots, u_{2 n} \in U, s_{1}, \cdots, s_{n} \in S, t_{1}, \cdots, t_{n-1} \in T$ such that

$$
\begin{aligned}
s & =s_{1} v_{1}, \quad w_{1}=w_{2} t, \\
s_{1} v_{2} & =s_{2} v_{3}, \quad w_{3} t_{1}=w_{4} t_{2}, \\
\cdots & \\
s_{n-1} v_{2 n-2} & =s_{n} v_{2 n-1}, \quad w_{2 n-1} t_{n-1}=w_{2 n}, \\
s_{n} v_{2 n} & =s^{\prime},
\end{aligned}
$$

and such that $s \neq s^{\prime}$. Let us suppose that $s, s^{\prime}, u_{1}, \cdots, t_{n-1}$ are chosen so that $n$ is as small as possible.

Lemma 3.4. Suppose that $\mu: S \rightarrow P$ is not a monomorphism and that (23) is the shortest set of factorisations exhibiting this fact. Then

(i) $u_{3} \notin u_{2} U^{1}$;

(ii) one or other of $u_{j-1}, u_{j+1}$ does not belong to $u_{j} U^{1}(j=3, \cdots, 2 n-2)$;

(iii) $u_{2 n-2} \notin u_{2 n-1} U^{1}$.

Proof. (i) If $u_{2} u=u_{3}\left(u \in U^{1}\right)$, then

$$
w_{3} t_{1}=w w_{2} t_{1}=w w_{1} \in W .
$$

Hence, if $s^{\prime \prime}=s_{2}\left(v v_{1}\right)$, we have

$$
\begin{aligned}
s^{\prime \prime} & =s_{2}\left(v v_{1}\right), \quad w w_{1}=w_{4} t_{2}, \\
s_{2} v_{4} & =s_{3} v_{5}, \quad \ldots w_{5} t_{2}=w_{6} t_{3}, \\
s_{n} v_{2 n} & =s^{\prime} .
\end{aligned}
$$

Thus $\mu\left(s^{\prime \prime}\right)=\mu\left(s^{\prime}\right)$, and the set of factorisations demonstrating the fact is shorter than the set (23). Hence $s^{\prime \prime}=s^{\prime}$. Now, either $u_{1}=u_{2}$ or $u_{2}$ is a potential divisor of $u_{1}$; for, if $u, u^{\prime} \in U^{1}$, then

$$
\begin{aligned}
u u_{2}=u^{\prime} u_{2} & \Rightarrow w w_{2}=w^{\prime} w_{2} \\
\Rightarrow w w w_{2} t_{1}=w^{\prime} w_{2} t_{1} & \Rightarrow w w_{1}=w^{\prime} w_{1} \Rightarrow u u_{1}=u^{\prime} u_{1} .
\end{aligned}
$$

Hence, since $U$ is division-ordered, there exists $u^{*} \in U^{1}$ such that $u^{*} u_{2}=u_{1}$. Hence

$$
s=s_{1} v_{1}=s_{1} v^{*} v_{2}=s_{2} v^{*} v_{3}=s_{2} v^{*} v v_{2}=s_{2} v v_{1}=s^{\prime \prime}
$$

and so $s=s^{\prime}$, contrary to hypothesis.

A similar argument establishes (iii). 
To prove (ii), suppose first that $j=2 i$, an even integer, and that

$$
u_{2 i-1}=u_{2 i} u^{\prime}, \quad u_{2 i+1}=u_{2 i} u^{\prime \prime},
$$

where $u^{\prime}, u^{\prime \prime} \in U^{1}$. Then the two rows

$$
\begin{aligned}
s_{i-1} v_{2 i-2} & =s_{i} v_{2 i-1}, \quad w_{2 i-1} t_{i-1}=w_{2 i} t_{i}, \\
s_{i} v_{2 i} & =s_{i+1} v_{2 i+1}, \quad w_{2 i+1} t_{i}=w_{2 i+2} t_{i+1}
\end{aligned}
$$

of the factorisations (23) can be replaced by the single row

where

$$
s_{i-1} v_{2 i-2}=s_{i+1} v^{*}, \quad w^{*} t_{i-1}=w_{2 i+2} t_{i+1},
$$

for

and

$$
u^{*}=u_{2 i-1} u^{\prime \prime}=u_{2 i} u^{\prime} u^{\prime \prime}=u_{2 i+1} u^{\prime}
$$

$$
s_{i-1} v_{2 i-2}=s_{i} v_{2 i-1}=s_{i} v_{2 i} v^{\prime}=s_{i+1} v_{2 i+1} v^{\prime}=s_{i+1} v^{*},
$$

$$
w^{*} t_{i-1}=w^{\prime \prime} w_{2 i-1} t_{i-1}=w^{\prime \prime} w_{2 i} t_{i}=w_{2 i+1} t_{i}=w_{2 i+2} t_{i+1} .
$$

The set of factorisations (23) can thus be shortened, contrary to hypothesis.

Suppose now that $j=2 i+1$, an odd integer, and that

$$
u_{2 i}=u_{2 i+1} u^{\prime}, \quad u_{2 i+2}=u_{2 i+1} u^{\prime \prime} .
$$

Then, by an argument very similar to that employed in the previous case, we can show that the three rows

$$
\begin{aligned}
s_{i-1} v_{2 i-2} & =s_{i} v_{2 i-1}, & w_{2 i-1} t_{i-1} & =w_{2 i} t_{i}, \\
s_{i} v_{2 i} & =s_{i+1} v_{2 i+1}, & w_{2 i+1} t_{i} & =w_{2 i+2} t_{i+1}, \\
s_{i+1} v_{2 i+2} & =s_{i+2} v_{2 i+3}, & w_{2 i+3} t_{i+1} & =w_{2 i+4} t_{i+2}
\end{aligned}
$$

of the factorisations (23) can be replaced by the two rows

$$
\begin{aligned}
& s_{i-1} v_{2 i-2}=s_{i} v_{2 i-1}, \quad w_{2 i-1} t_{i-1}=w^{*} t_{i+1}, \\
& s_{i} v^{*}=s_{i+2} v_{2 i+3}, \quad w_{2 i+3} t_{i+1}=w_{2 i+4} t_{i+2},
\end{aligned}
$$

where $u^{*}=u^{\prime} u_{2 i+2}=u^{\prime} u^{\prime \prime} u_{2 i+1}=u^{\prime \prime} u_{2 i}$. Thus again we obtain a reduction in the length of the factorisations (23), contrary to hypothesis. This completes the proof of the lemma.

Returning now to the proof of the theorem, we consider the factorisations (23). Since $U$ is totally division-ordered and since, by Lemma 3.4 (i), $u_{3}$ is neither equal to nor divisible by $u_{2}$, we must have that $u_{2}$ is divisible by $u_{3}$; hence, by Lemma 3.4 (ii), $u_{4}$ is neither equal to nor divisible by $u_{3}$, and so $u_{3}$ is divisible by $u_{4}$. Hence, by Lemma 3.4 (ii), $u_{5}$ is neither equal to nor divisible by $u_{4}$, and so $u_{4}$ is divisible by $u_{5}$. Continuing in this way, we end with the statement that $u_{2 n-2}$ is divisible by $u_{2 n-1}$, contrary to Lemma 3.4 (iii). Hence $\mu$ must in fact be one-one. 
A similar argument shows that $v$ is one-one.

To establish the intersection property, suppose by way of contradiction that there exist $s \in S, t \in T$ such that $\mu(s)=v(t)$, but $s \notin V$. Then there exist $u_{1}, \cdots, u_{2 n-1} \in U, s_{1}, \cdots, s_{n-1} \in S, t_{1}, \cdots, t_{n-1} \in T$ such that

$$
\begin{gathered}
s=s_{1} v_{1}, \quad w_{1}=w_{2} t_{1}, \\
s_{1} v_{2}=s_{2} v_{3}, \quad w_{3} t_{1}=w_{4} t_{2}, \\
\cdots \\
s_{n-2} v_{2 n-4}=s_{n-1} v_{2 n-3}, \quad w_{2 n-3} t_{n-2}=w_{2 n-2} t_{n-1}, \\
s_{n-1} v_{2 n-2}=v_{2 n-1}, \quad w_{2 n-1} t_{n-1}=t,
\end{gathered}
$$

and again we can suppose that $s, t, u_{1}, \cdots, t_{n-1}$ are chosen so that $n$ is as small as possible.

Closely analogous to Lemma $\mathbf{3 . 4}$, we have

Lemma 3.5. Suppose that $\mu(S) \cap v(T)$ properly contains $\mu \varphi(U)$ and that (24) is the shortest set of factorisations exhibiting this fact. Then

(i) $u_{3} \notin u_{2} U^{1}$;

(ii) one or other of $u_{j-1}, u_{j+1}$ does not belong to $u_{j} U^{1}(j=3, \cdots, 2 n-3)$;

(iii) $u_{2 n-3} \notin u_{2 n-2} U^{1}$.

Proof. The proof of (ii) is identical to the proof of Lemma 3.4 (ii). The proofs of (i) and (iii) are very similar. Considering (iii) by way of illustration, we suppose that $u_{2 n-3}=u_{2 n-2} u$, where $u \in U^{1}$. Then

$$
s_{n-2} v_{2 n-4}=s_{n-1} v_{2 n-3}=s_{n-1} v_{2 n-2} v=v_{2 n-1} v
$$

and so if we replace the last two lines of the factorisations (24) by the single line

$$
s_{n-2} v_{2 n-4}=v_{2 n-1} v, \quad w_{2 n-1} w t_{n-2}=t^{\prime},
$$

we obtain a set of factorisations which asserts that $\mu(s)=v\left(t^{\prime}\right)$.

Hence $s \in V$ by the minimal property of (24) - a contradiction.

It is now easy to use the totally division-ordered property of $U$ to derive a contradiction from the factorisations (24), in exactly the same way as for the factorisations (23).

One corollary of Theorem 3.3 seems interesting enough to deserve a mention. It is shown in the proof of ([4], Corollary 2.7) that finite monogenic (cyclic, in the terminology of $[\mathbf{l}]$ ) semigroups are totally division-ordered. Hence

CoRollary 3.6. Finite monogenic semigroups are CSg-amalgamable. 


\section{References}

[1] A. H. Clifford and G. B. Preston, The algebraic theory of semigroups, vol. 1, Math. Surveys of the American Math. Soc. 7 (Providence, 1961).

[2] Peter Freyd, Abelian categories (Harper and Row, New York, 1964).

[3] J. M. Howie. 'The embedding of semigroup amalgams', Quart. J. Math. Oxford 15 (1964), $55-68$.

[4] J. M. Howie and J. R. Isbell, 'Epimorphisms and dominions. II', J. Algebra 6 (1967), $7-21$.

[5] John R. Isbell, 'Epimorphisms and dominions', Proc. Conference on Categorial Algebra La Jolla 1965 (Springer-Verlag, Berlin, 1966).

[6] Barry Mitchell, Theory of categories (Academic Press, New York, 1965).

[7] B. H. Neumann, 'An essay on free products of groups with amalgamations', Phil. Trans. Roy. Soc. A 246 (1954), 503--554.

University of Stirling

Stirling

Scotland 